\title{
Networked Pantheon: a Relational Database of Globally Famous People
}

\author{
Pablo Beytía ${ }^{1}$ and Janosch Schobin ${ }^{2}$ \\ Preprint citation: Beytía, P., \& Schobin, J. (2018). Networked Pantheon: a Relational Database \\ of Globally Famous People. Retrieved from osf.io/8xm46
}

\begin{abstract}
This article presents Networked Pantheon, a relational database of biographies of globally famous people spanning the last 5,500 years of human history. This source of information is intended to complement Pantheon 1.0 (Yu et. al. 2016), a biographical dataset that includes temporal, spatial, gender, and occupational information on 11,341 world-renowned people defined as those who have their biographies in 25 or more Wikipedia language-versions-. Networked Pantheon adds information about the biographical links between these historical figures, which was compiled from the hyperlinks between the biographies in English Wikipedia. This digital method allows technics from network analysis to be used to study the relationships between globally famous people, and thus to calculate different measures of historical centrality for individuals, cities, countries, genders, and occupations. Networked Pantheon complements the historical popularity indicators of Pantheon 1.0 with measurements of the centrality of the figures in the network of biographical references, allowing for an approximation to the information flows between different territories, genders, and occupations of famous people over time.
\end{abstract}

Keywords: world history, Wikipedia, digital sociology, digital humanities, network analysis

\section{Introduction}

In recent years there has been a growing scientific interest in the organization of knowledge stored in Wikipedia. On the one hand, the information structured in this digital encyclopedia has begun to be used as an input to study interesting phenomena, such as historical trends (Schich et al 2014, Jara-Figueroa et al 2015, Reznik \& Shatalov 2016, Menini et al 2017), the structure of links between different languages (Mehler et al 2011, Aragon et al 2012, Eom et al 2014, Ronen et al 2014, Ban et al 2017), or the geopolitical instability (Apic and Rusell 2011). On the other hand, this information has been used to understand the 'systemic biases' of Wikipedia's collective repository, emphasizing differences in the degree of information on different territories (Graham 2015, Roll et al 2016), cultures (Overell and Rüger 2011, Nemoto and Gloor 2011, Eom et al 2014) and genders (Gruwell 2015, Shane-Simpson and Gillespie-Lynch 2017).

\footnotetext{
${ }^{1}$ Department of Social Sciences, Humboldt-Universität zu Berlin.

2 Department of Sociology, Universität Kassel.
} 
Following a series of pioneering studies in the area (Murray 2003, Popescu et al 2010, Michel et al 2011, Skiena and Ward 2013, Schich 2014), a privileged database to conduct research of both types was published in 2016. It is Pantheon 1.0, a dataset of globally famous people that includes information about the 11,341 biographies present in 25 or more language-versions of Wikipedia (Yu et al 2016). Using the number of languages in which each biography is available as a proxy for its global cultural relevance, this dataset not only brings together indispensable information for historical research on recognized personalities (such as year, city and geographic coordinates of birth), but also includes information on the gender and occupation of each of them and metrics on their historical popularity. In this way, Pantheon data allows the study of the organization of the world's biographical knowledge in Wikipedia in a multidimensional way, facilitating the study of temporal, spatial, gender and occupational aspects in an enormous range of time $(3,500 \mathrm{BC}-2005 \mathrm{AC})$.

This article presents Networked Pantheon, a relational database that has been designed to complement Pantheon 1.0. Mainly, this data source provides information on the biographical relationships between the historical figures, approaching these links from the hyperlinks between the Wikipedia biographies in English. This method, which has been used in previous research (Aragon et al 2012, Skiena and Ward 2013), allows the study of Pantheon 1.0 biographies using methodologies from network analysis. Added to this, Networked Pantheon adds data on the year of death of each historical character, allowing the lifespan of each recorded individual to be known. With this information it is possible, for example, to assess whether the demographic characteristics of this sample of famous people are related to actual demographic processes recorded in history (cf. Reznik \& Shatalov 2016). Fundamentally, however, the dates of death make it possible to simulate with greater precision the evolution of the biographical relations between notable characters over time, which could be used to better understand the collective structuring of historical knowledge in Wikipedia, as well as to advance in the study of the dynamic properties of temporal networks (Li et al 2017).

\section{Methods}

\subsection{Link Data}

The hyperlinks between the Wikipedia articles of the biographies of 11,340 notorious personae of history contained in the original Pantheon Database were extracted using the $\mathrm{R}$ Packages rvest (Wickham, 2016) and stringi (Gagolewski et al 2015). These hyperlinks were obtained from the English Wikipedia articles. This language version was chosen because it is the most complete version -those with more articles, biographies, editions, and editors (Nemoto and Gloor 2011, Aragon et al 2012)- and also the one that registers the largest number of historical figures with biographies in 25 or more different languages. English Wikipedia includes a biography for all but one of the famous individuals recorded in Pantheon $1.0^{3}(11,340$ people in total), followed by the French $(11,334)$, German $(11,319)$, Russian $(11,314)$ and Spanish $(11,287)$ versions. While it is expected that the selection of language implies a linguistic bias getting a better record of relations between Englishspeaking figures (and by extension those of geographic sectors close to English speaking countries), it has also been documented that a great part of the links indicated in Wikipedia

3 The only biography included in Pantheon, but not available in English Wikipedia, is that of the Italian photographer Augusto de Luca. 
biographies overcome linguistic barriers (Aragon et al 2012), and this phenomenon should be more common when dealing with famous historical figures recorded in many different languages.

It has to be noted that in order to extract the links it was necessary to update de URL of the pages of the famous personalities. This was done in a two-stage process. First, we checked if the term "disambiguation" appeared in the content of the page. (To be more precise, if it appeared more than 3 times, because a disambiguation page of Wikipedia contains at least four times the word "disambiguation"). If this was the case $(\mathrm{N}=235)$ we checked the page by hand and worked out the current URL by following the suggested links on the disambiguation page. In a second step, we extracted the html code from the URL of the corrected Dataset. Another 44 URLs showed connection errors. We corrected these by searching for the names of the respective famous personalities on the Wikipedia page and identifying them from the features available in Pantheon 1.0 (year of birth, city of birth, gender and main occupation).

Finally, we extracted 126,279 links present in the Wikipedia pages of 11,340 famous individuals. The date of extraction was the 16.04.2018.

\subsection{Year of death}

We extracted the year of death from the html code of the Wikipedia pages of the famous personalities by searching for the string "died" in connection with various types of dates. This search was conducted by matching the html data to various regular expression that represented typical types of dates. It has to be noted that there are many different types of dates present in Wikipedia biography pages (e.g. 340 BC; 51 b.c.; 18 April 1955; July 18, 2001, and many more). The extracted dates were checked for plausibility by comparing them with the birthdates already present in the original Pantheon database. This way we were able to extract the year of death of all but 436 cases. The later we searched by hand in English Wikipedia, finding the information for 300 cases (extracted on 16.04.2018). Some biographies just did not have a date of death, mostly because they are very old historical figures that lack precise historical records. These biographies together total 136 cases, whose dates of death were imputed. These imputations were calculated from the median age span associated with the historical period of each figure, which was approximated from the age span of the 10 closest cases according to the year of birth.

\subsection{Centrality measures}

To facilitate the statistical use of relational indicators, Networked Pantheon includes for each figure registered in the database a series of structural measures that were calculated from the network of biographical connections. These indicators are the following:

- Degree: the number of connections or edges that one node (a biography) has to other nodes (Freeman 1979).

- Indegree: the number of edges (hyperlinks) going into a node.

- Outdegree: the number of edges coming out of a node.

- EigenCentrality (eigenvector): it measures the centrality of an actor as proportional to the sum of the centralities of its neighbors in the graph. It was calculated with an iteration of 100 (Bonacich 1987).

- PageRank: is a measure of the global importance of nodes, computed recursively by putting a greater weight on incoming connections from central nodes (Brin and Page 
1998, Page et al 1999). It was calculated with an epsilon of 0.001 and a probability of 0.85 .

- Eccentricity: distance between a node and the node furthest away from it in the network (Hage and Harary 1995).

- ClosenessCentrality: an indicator of the distance between a node and all the other nodes in the network, based on the arithmetic mean of the minimum path between the nodes (Freeman 1979).

- HarmonicClosenessCentrality: an indicator of the distance between a node and all other nodes in the network, based on the harmonic mean of the minimum path between the nodes (Rochat 2009).

- BetweenessCentrality: frequency in which a node appears in the shortest path between the nodes of the network (Freeman 1970, Brandes 2001).

- Authority: the degree to which a webpage (a biography) is pointed to by many good hubs (Kleinberg 1998).

- Hub: the degree to which a webpage (a biography) points to many good authorities (Kleinberg 1998).

- Clustering: the degree to which nodes tend to cluster together (Saramäki et al 2007).

\subsection{Biographical Centrality Index (BCI)}

As a complement to the Historical Popularity Index (Yu et al 2016), here we include a Biographical Centrality Index (BCI) that weighs, for each historical figure, its cultural ubiquity (approximated by the number of languages in which a biography is available) and its biographical connectivity (approximated by the PageRank algorithm). Considering the number of languages of a biography (NL) and its PageRank (PR), the BCI of a famous character is summarized in the following formula:

$$
\mathrm{BCI}=\frac{\left(\mathrm{NL}^{*} \mathrm{PR}\right)-\left(\mathrm{NL}^{*} \mathrm{PR}\right) \min }{(\mathrm{NL} * \mathrm{PR}) \max -(\mathrm{NL} * \mathrm{PR}) \min }
$$

The BCI can be understood as a standardized indicator (with Feature Scaling method) of the probability that a historical character will appear linked to a random biographical search in a random language on Wikipedia. It refers to the degree of exposure that a figure has in different languages, weighing it by its centrality (PageRank) in the network of biographical references of Wikipedia in English. It is an indicator relating to the distribution of the cultural ubiquity and biographical connectivity of the total number of characters considered, and can be interpreted as the proportion of centrality of a biography in comparison with the most central biography of the sample (in a value between 0 and 1, where 1 represents the greatest possible centrality).

BCI must be rigorously distinguished from the Historical Popularity Index (HPI) included in Pantheon 1.0 (Yu et al 2016) for at least three reasons:

1. BCI considers biographical connectivity as a relevant indicator for ranking the influence of characters on the discursive structure of Wikipedia.

2. It does not consider the 'demand' for information on historical figures ('pageViews' variable), but focuses on describing the structure of the production and supply of information.

3. It is not intended to be an adequate indicator for understanding the historical importance of each character -which should consider, for example, an adjustment to 
regulate the excessive relative importance of some 20th century characters- but rather is intended to be a tool for understanding how historical memory is currently being structured in Wikipedia, highlighting the disparities in the distribution of historical information and the concentrations of reference flows in certain periods, territories, genders, and occupations.

\subsection{Year of birth}

This data was already present in the original Pantheon database. However, we corrected a total of 9 cases that had wrong birthdates that came up while we searched for the Year of Death of the 436 cases we extracted manually.

\subsection{Recoding occupational dimensions}

Due to the independent historical relevance of religious, military and governmental occupations, we create a new variable of the occupational domain ("domain2"), which separate the "Institutions" dimension in three different dimensions: Government, Religion and Army (see annex for details).

\section{Database description}

\subsection{General aspects}

Networked Pantheon database records 126,279 direct relationships between 11,340 web biographies of globally famous people, defined as those who have Wikipedia biographies in more than 25 different languages. On average, these biographies have 11.12 hyperlinks to other biographies, ranging from 0 (e.g., Al Capone) to 105 (Meryl Streep). The average number of hyperlinks incoming to the biographies is also 11.12 and varies between 0 (e.g. Josep Guardiola) and 414 (Barack Obama). The diameter of the network -that is, the largest distance between two pairs of nodes- is 15 , while the average path length is 4.81 .

Figure 1 illustrates the general topography of the network in three different ways: applying a distribution algorithm (A), using a distribution algorithm and colors to distinguish large occupational domains (B), and associating the nodes and edges to the geographic location of birth and to an occupational domain (C).

\section{Graph 1. Distributions of the network structure}
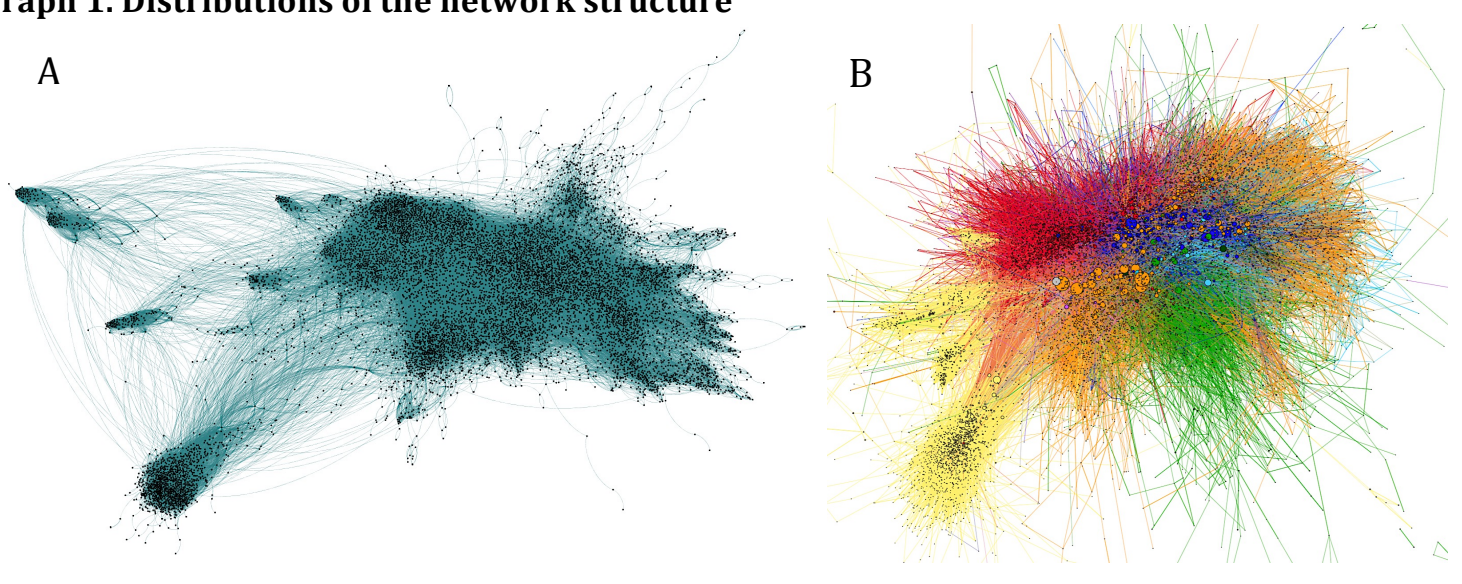


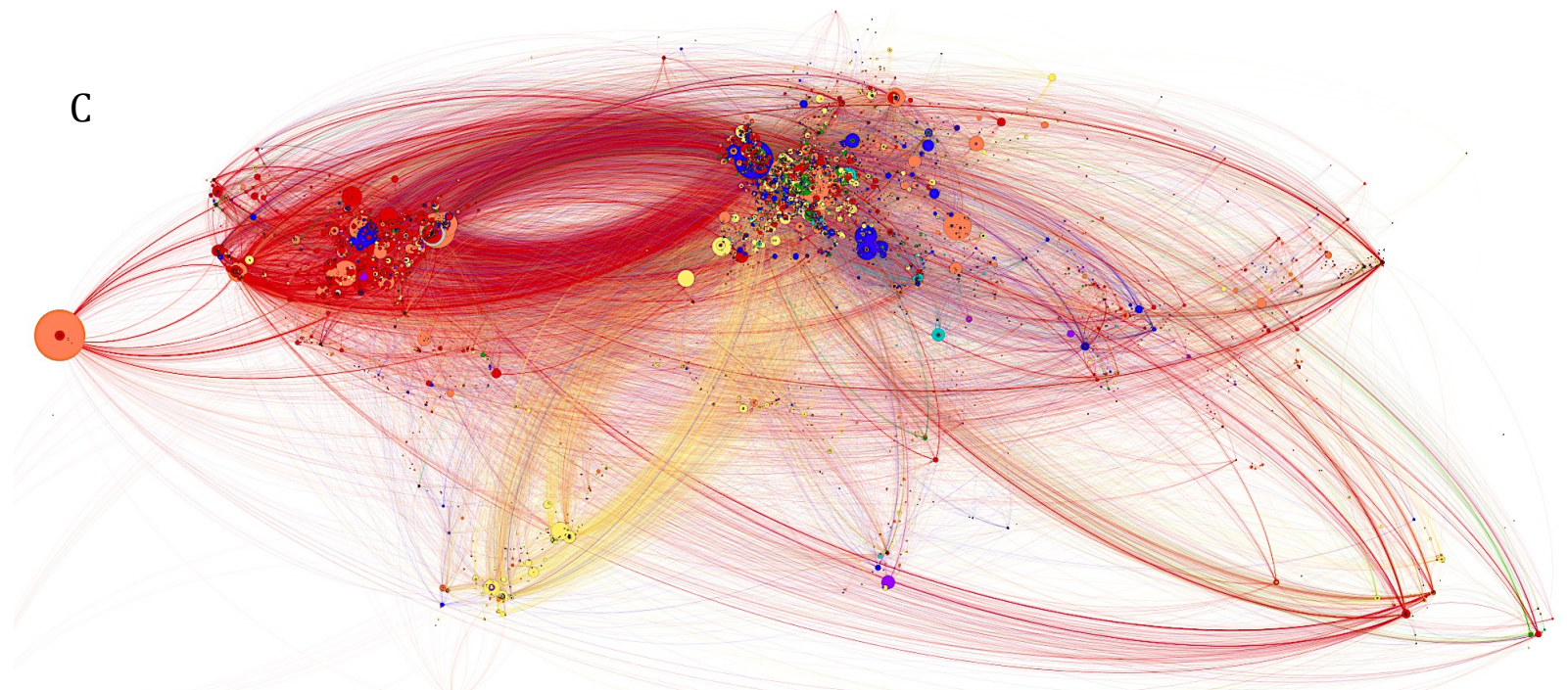

Note: A) Networked Pantheon distributed using Force Atlas 2 algorithm, B) Yifan Hu distribution differentiating colors by occupational domain, C) geographical distribution of links differentiating colors by occupational domain and node size by PageRank algorithm.

\subsection{Dynamic analysis}

This database allows diachronically researching the network of biographical references and comparing the structure of the historical record on specific periods. Figure 2 shows an example of the structural variation of the network over time, distinguishing a period in which the archaic civilizations emerged (A), another associated with the rise of the Greco-Roman world (B), a third epoch that is commonly referred to as the Middle Ages (C) and the period after the spread of the printing press and the beginning of globalization (D). The colors of the nodes represent broad occupational domains (politics, religion, art, humanities, science and technology, army, sports, business and law, public figure and exploration). As can be seen, these are completely different networks according to their shape, size, density and occupational composition, which can be analyzed independently for specialized studies.

\section{Graph 2. Example of temporal dynamics}

A

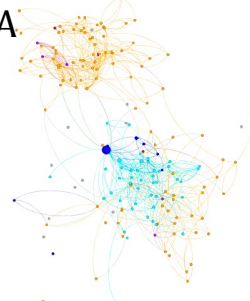

$3,500 \mathrm{BC}-651 \mathrm{BC}$
$\mathrm{B}$

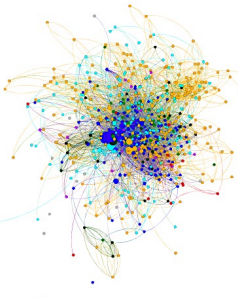

$650 \mathrm{BC}-476 \mathrm{AC}$
C

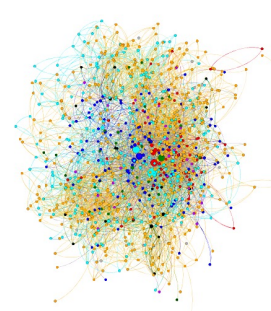

477 AC - 1491

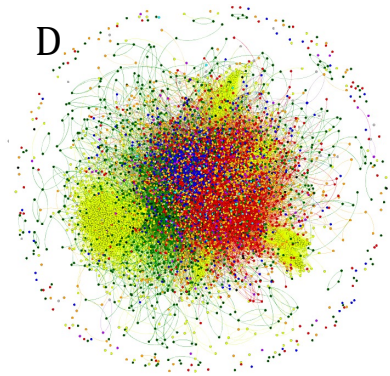

$1492-2005$

Note: The colors represent wide occupational domains approximated using the variable domain2 (see appendix)

\subsection{Flows of biographical references}

On the other hand, with the information of Networked Pantheon biographical relations between historical figures can be geographically located, and with it is possible to approach 
the flows of informative links between different regions of the world. Map 1 displays the worldwide distribution of biographical references, showing a wide concentration of links between Western Europe and the United States.

\section{Map. 1. Geographical distribution of biographical reference flows}

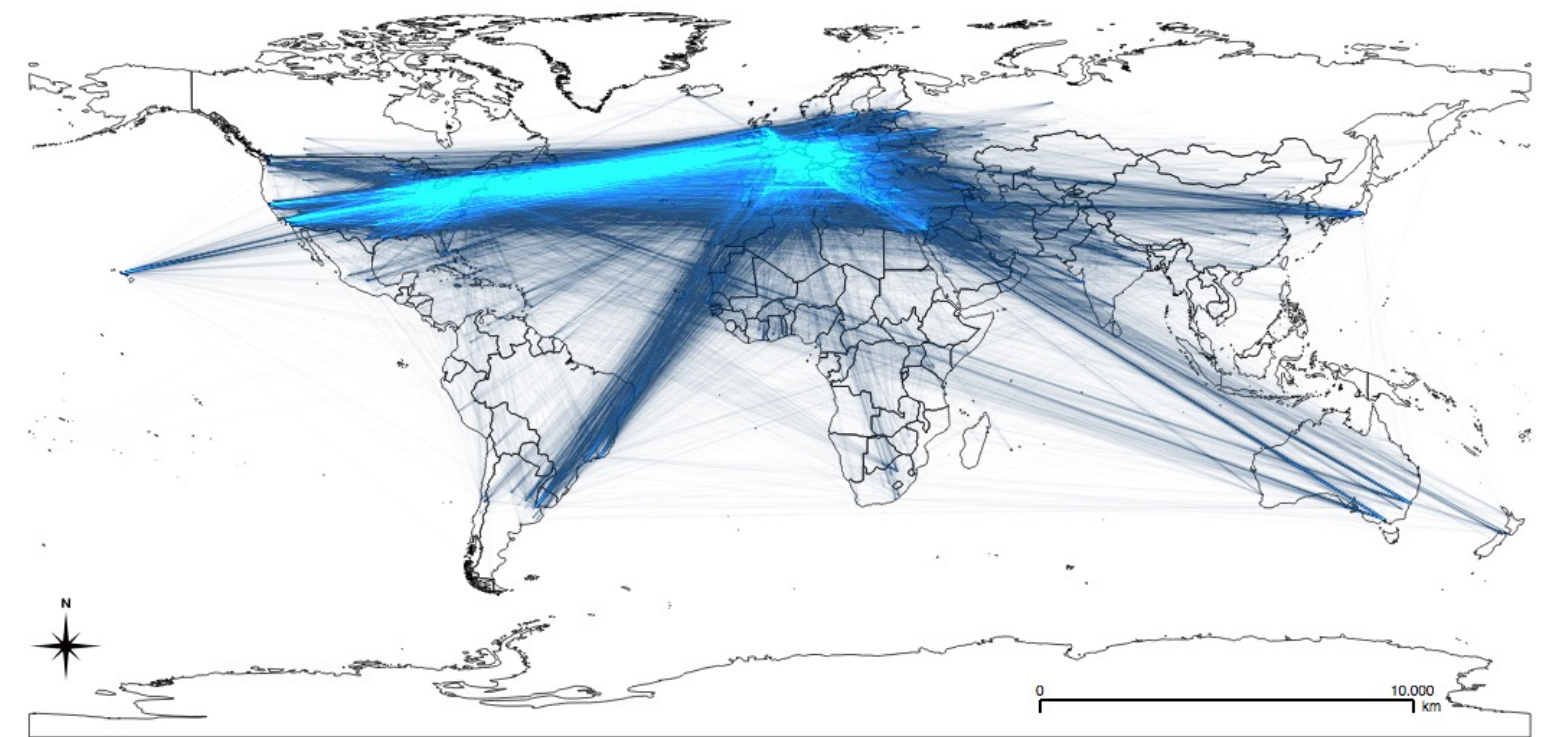

Note: The position of each node is defined by the place of birth.

In addition, these flows of references can be observed according to different criteria -such as the place of birth, occupation or gender of historical figures-. For example, graph 3 represents the (origin and destination) flows of references between different occupational domains, showing a high level of professional self-referencing: references to people in an occupational domain usually come from biographies of people in the same occupational domain. 
Graph 3. Flows of biographical references between different occupational fields.

Source domains

Target domains

ARTS $(35.9 \%)$

ARTS (32.5\%)

GOVERNMENT (19\%)

GOVERNMENT (22.2\%)

SPORTS $(18.8 \%)$

SPORTS (17.8\%)

HUMANITIES (11.9\%)

HUMANITIES (13.6\%)

SCIENCE \& TECHNOLOGY (6\%)

SCIENCE \& TECHNOLOGY (5.6\%)

| RELIGION (3.5\%)

I PUBLIC FIGURE $(2.6 \%)$

- ARMY (1.7\%)

- BUSINESS \& LAW $(0.7 \%)$

- EXPLORATION $(0.4 \%)$

Note: on the left are the occupational domains ordered according to the number of biographical references (hyperlinks) they generate -specifying the percentage of the total in parentheses- and on the right are the same domains ordered according to the percentage of references they receive.

\subsection{Structural measures of centrality and influence}

The measures of historical popularity of Pantheon 1.0 -number of languages of a biography (numLangs), visits to the page of each biography on the Internet (PageViews) and Historical Popularity Index (HPI)- are complemented here with various structural measures of centrality and influence on the network of biographical references (Indegree, Betweenness, Closeness, Eigenvector and Pagerank, among others). Figure 4 shows the correlations between all of these measures, whether they are of historical popularity or biographical centrality. As can be seen, the HPI has a low correlation with Networked Pantheon's biographical centrality measures, some of which are highly interlinked with each other (Degree, Indegree, Eigenvector, PageRank, and Betweenness). The Biographical Centrality Index (BCI) is also quite correlated with this group of coefficients. Other measures (Closeness, Authority, Clustering, and Eccentricity) show considerable autonomy with respect to the other centrality coefficients.

Table 1 shows the comparison between the top 10 historical figures following some dissimilar biographical centrality coefficients. As can be seen, if the Eigenvector is used as a reference, 7 USA presidents would be among the 10 most influential characters, while the Authority coefficient grants an enormous relevance to ATP tennis players (who occupy the first 141 positions in the ranking). The PageRank, by contrast, tends to highlight the role of politicians of the last century and great humanists (Plato, Aristotle, Shakespeare), while the Betweenness considerably broadens the range of occupations, highlighting religious leaders 
(Pope John Paul II), ancient politicians (Charlemagne), scientists (Albert Einstein), sportsmen (Pelé) and businessmen (Roman Abramovich). The Closeness coefficient is the least Westernbiased, including a mixture of sultans, Nobel Prize winners in physics, Ukrainian athletes and politicians from Latvia or Benin.

\section{Graph 4. Correlation diagram between historical popularity and biographical centrality measures.}

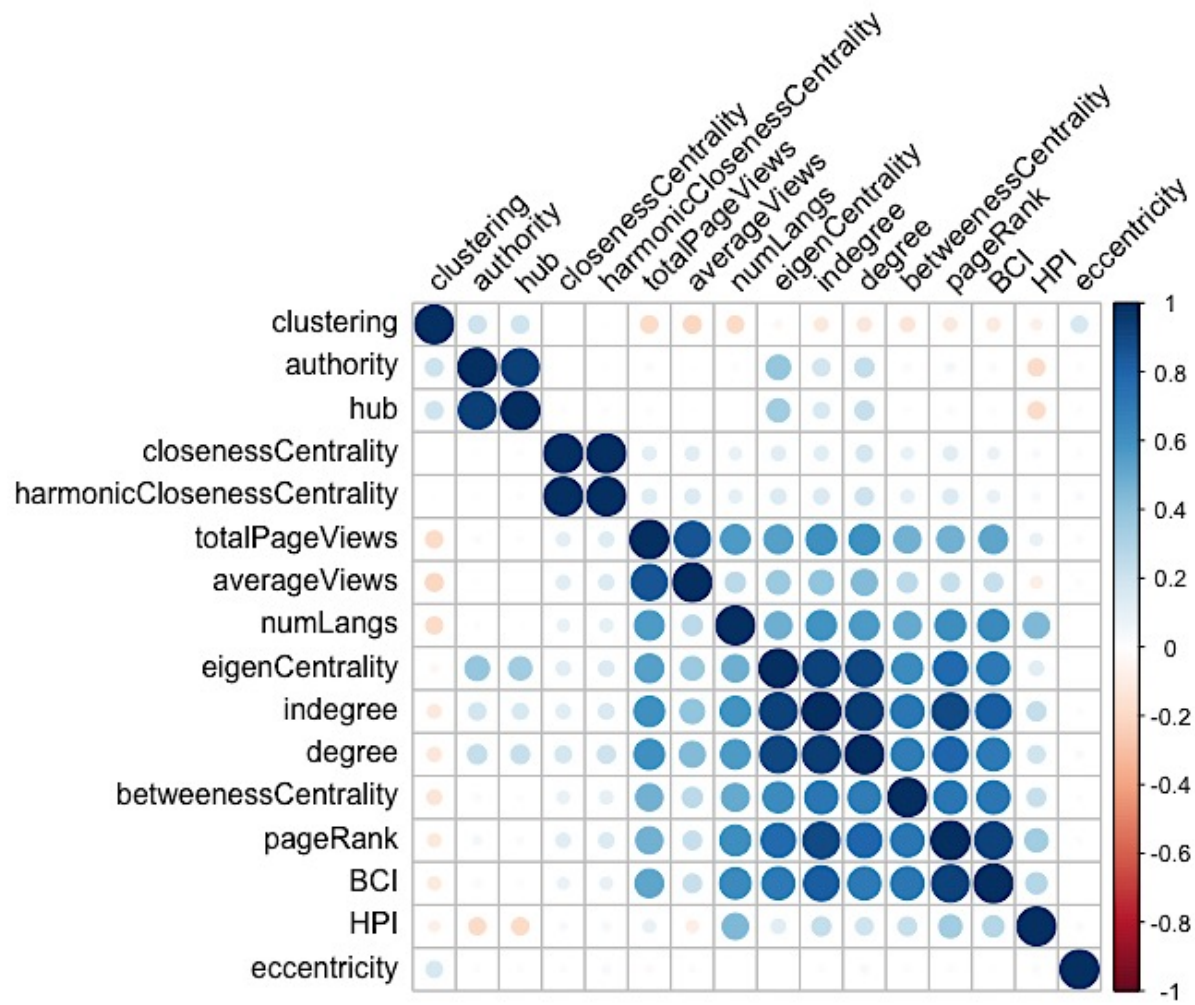

Note: circles denote statistically significant relationships. The larger the circle, the greater the relationship between two indicators of centrality or popularity. Blue tones denote positive relationships and red tones negative ones.

Table 1. Comparison between centrality measures (top 10 ranking)

\begin{tabular}{|l|l|l|l|l|l|l|}
\hline Rank & Betweenness & \multicolumn{1}{|c|}{ Closeness } & \multicolumn{1}{|c|}{ Authority } & \multicolumn{1}{|c|}{ Eigenvector } & \multicolumn{1}{|c|}{ PageRank } & BCI \\
\hline $\mathbf{1}$ & $\begin{array}{l}\text { Pope John Paul } \\
\text { II }\end{array}$ & $\begin{array}{l}\text { Mohammed V of } \\
\text { Morocco }\end{array}$ & Roger Federer & Barack Obama & Adolf Hitler & Barack Obama \\
\hline $\mathbf{2}$ & Adolf Hitler & $\begin{array}{l}\text { Hassan II of } \\
\text { Morocco }\end{array}$ & Rafael Nadal & George W. Bush & Barack Obama & Adolf Hitler \\
\hline $\mathbf{3}$ & Charlemagne & Eric Allin Cornell & Novak Djokovic & Ronald Reagan & George Bush & $\begin{array}{l}\text { William } \\
\text { Shakespeare }\end{array}$ \\
\hline $\mathbf{4}$ & George Bush & Carl Wieman & Andrew Murray & $\begin{array}{l}\text { William } \\
\text { Shakespeare }\end{array}$ & $\begin{array}{l}\text { William } \\
\text { Shakespeare }\end{array}$ & George W. Bush \\
\hline $\mathbf{5}$ & $\begin{array}{l}\text { Benito } \\
\text { Mussolini }\end{array}$ & Willis Lamb & Andy Roddick & Adolf Hitler & Joseph Stalin & Joseph Stalin \\
\hline $\mathbf{6}$ & Albert Einstein & Oleg Blokhin & Tomáš Berdych & Bill Clinton & Ronald Reagan & Aristotle \\
\hline $\mathbf{7}$ & Vladimir Putin & Valdis Zatlers & $\begin{array}{l}\text { Jo-Wilfried } \\
\text { Tsonga }\end{array}$ & Donald Trump & Plato & Plato \\
\hline $\mathbf{8}$ & Pelé & Igor Belanov & $\begin{array}{l}\text { Nikolay } \\
\text { Davydenko }\end{array}$ & John F. Kennedy & Bill Clinton & Karl Marx \\
\hline $\mathbf{9}$ & Ronald Reagan & Mathieu Kérékou & David Ferrer & Roger Ebert & $\begin{array}{l}\text { Winston } \\
\text { Churchill }\end{array}$ & Isaac Newton \\
\hline $\mathbf{1 0}$ & $\begin{array}{l}\text { Roman } \\
\text { Abramovich }\end{array}$ & $\begin{array}{l}\text { Raymond Davis } \\
\text { Jr. }\end{array}$ & James Blake & Richard Nixon & Aristotle & Albert Einstein \\
\hline
\end{tabular}




\subsection{Biographical Centrality Index (BCI)}

The BCI is an indicator of the positioning of biographical information on Wikipedia, considering its dissemination in multiple languages and its centrality in the network of references (hyperlinks). As can be seen from map 2, the biographical centrality in Wikipedia is not evenly distributed across the territory but is clearly concentrated in the United States and Western Europe. In addition, the biographical positioning is concentrated on certain historical periods. Given that the general record of biographies has grown exponentially over the last five centuries (probably due to technological evolution and media spread), there is also a greater accumulation of biographical centrality in that period: when recorded famous people increase, the possibilities of biographical relationships also increase (Figure 5, A). For that reason, it is more interesting to note the changes in the average of biographical centrality over time. Figure 5 (B) shows that there are several periods in ancient history when celebrities on average acquired a wide resonance and influence in the current historical discourse. For example, a period around $400 \mathrm{BC}$, where Greece was a center of science and humanities, or around the year 0 , where there was a boom in religious figures linked to Christianity.

Map 2: Geographical concentration: cumulative sum by country and place of birth of more central characters

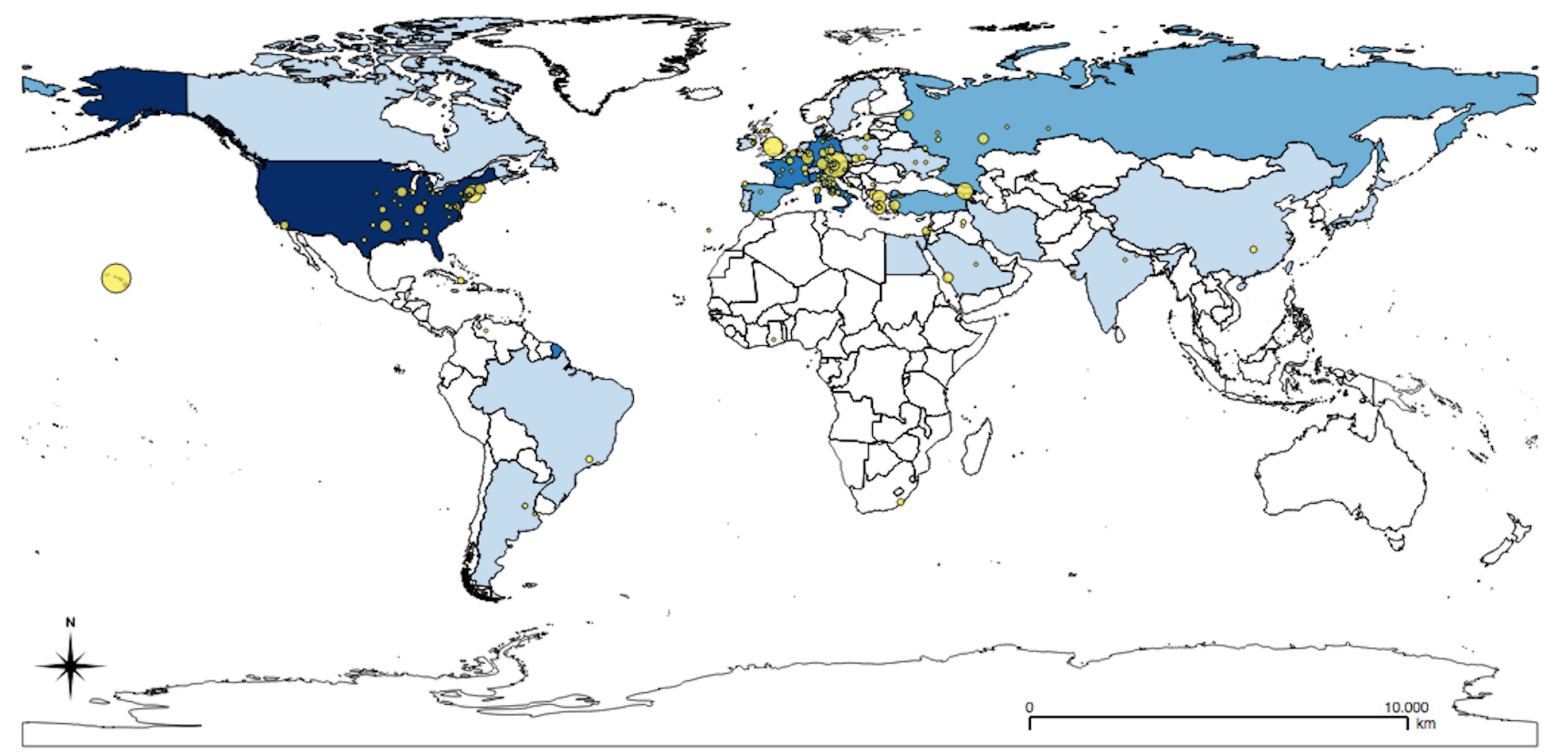

Note: color represents the total sum of Biographical Centrality Index for each country: the darker the color, the greater the accumulation of BCI. The yellow circles are pointing to the birthplace of people with high BCI (over 2 standard deviations).

Graph 5. Historical concentration: variation of the BCI sum and average

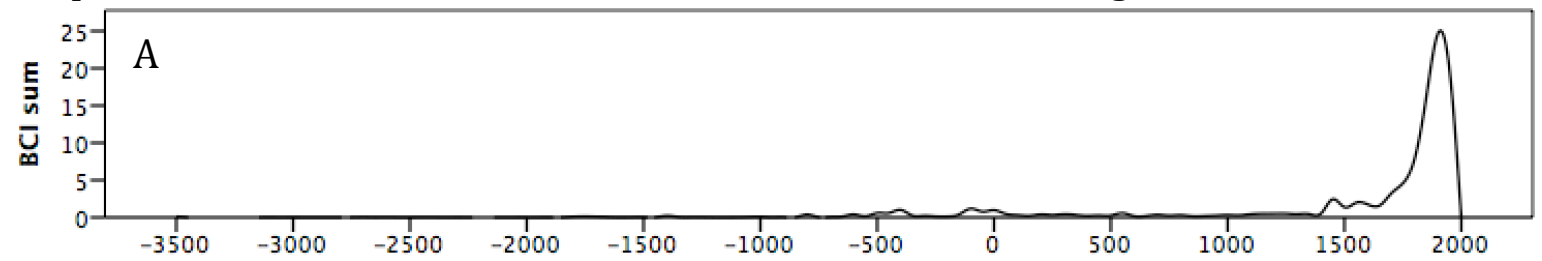




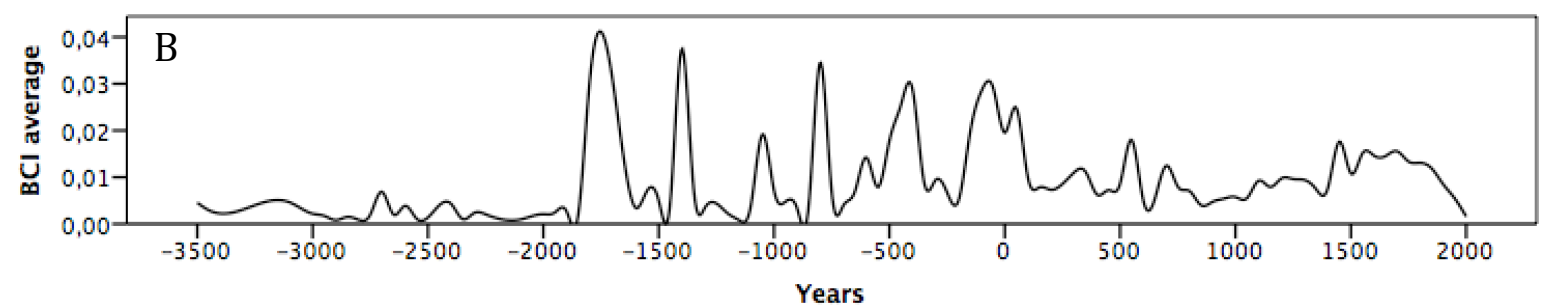

Note: The historical average of BCI was calculated for the accumulation of historical characters over periods of 50 years.

\section{Possible uses and research fields}

Given the technical characteristics and examples described in this article, Networked Pantheon could be used for a variety of purposes, such as, for example:

- Approaching the historical links between globally famous people (or the collective memory we currently have of these links).

- Using these biographical relationships as a proxy to investigate the cultural exchange between different cities, countries or continents.

- To model the network of biographical relationships between historical figures through space, as well as to simulate their evolution through time.

- Calculate and compare historical centrality indicators for each famous individual, city, country, continent, gender or occupation included in the database.

- Analyze flows of biographical references between people from different cities, countries, continents, genders or occupations.

In this way, this database aims to increase the huge analytical potential of Pantheon 1.0, adding relational observations that could be used to answer new questions about digitally constructed history, collaborative media, cultural influence, distribution of information, global collective memory and biographical knowledge structuration, among other areas.

These topics should not only be associated with widely established fields of research -such as computer science, media studies, computational linguistic or digital humanities- but also with the emerging computational and digital social sciences (Lazer et al 2009), which in recent years have achieved an adequate level of methodological reflection (Rogers 2013; Schäfer and van Es 2017; Venturini et al 2018) and a growing institutionalization in new sub disciplines -such as digital sociology (Orton-Johnson and Prior 2013; Lupton 2014; Marres 2017), digital anthropology (Miller and Slater 2000; Horst and Miller 2013), digital geography (Zook et al 2004; Graham 2014) or social physics (Pentland 2010 and 2014)-. 


\section{References}

Apic, G., Betts, M. J., \& Russell, R. B. (2011). Content disputes in Wikipedia reflect geopolitical instability. PloS one, 6(6), e20902.

Aragon, P., Laniado, D., Kaltenbrunner, A., \& Volkovich, Y. (2012). Biographical social networks on Wikipedia: a cross-cultural study of links that made history. In Proceedings of the eighth annual international symposium on Wikis and open collaboration (p. 19). ACM.

Ban, K., Perc, M., \& Levnajić, Z. (2017). Robust clustering of languages across Wikipedia growth. Royal Society open science, 4(10), 171217.

Brandes, U. (2001). A faster algorithm for betweenness centrality. Journal of mathematical sociology, 25(2), 163-177.

Brin, S., \& Page, L. (1998). The anatomy of a large-scale hypertextual web search engine. Computer networks and ISDN systems, 30(1-7), 107-117.

Bonacich, P. (1987). Power and centrality: A family of measures. American journal of sociology, 92(5), 1170-1182.

Eom, Y. H., Aragón, P., Laniado, D., Kaltenbrunner, A., Vigna, S., \& Shepelyansky, D. L. (2015). Interactions of cultures and top people of Wikipedia from ranking of 24 language editions. PloS one, 10(3), e0114825.

Freeman, L.C. (1978). Centrality in social networks. Conceptual clarification. Social Networks, 1: 215-239.

Gagolewski, M., Tartanus, B., Keyes, O., Bujarski, M. P., \& Adler, A. (2015). Stringi: Zenodo.

Graham, M. (2014). Internet geographies: Data shadows and digital divisions of labour. In Society and the Internet: How Networks of Information and Communication are Changing our Lives, eds M. Graham and W. H. Dutton. Oxford: Oxford University Press. 99-116.

Graham, M., Straumann, R. K., \& Hogan, B. (2015). Digital divisions of labor and informational magnetism: Mapping participation in Wikipedia. Annals of the Association of American Geographers, 105(6), 1158-1178.

Gruwell, L. (2015). Wikipedia's Politics of Exclusion: Gender, Epistemology, and Feminist Rhetorical (In) action. Computers and Composition, 37, 117-131.

Hage, P., \& Harary, F. (1995). Eccentricity and centrality in networks. Social networks, 17(1), 57-63.

Horst, H. A., \& Miller, D. (Eds.). (2013). Digital anthropology. A\&C Black.

Jara-Figueroa, C., Yu, A. Z., \& Hidalgo, C. A. (2015). The medium is the memory: how communication technologies shape what we remember. arXiv preprint:1512.05020.

Kleinberg, J. M. (1998). Authoritative sources in a hyperlinked environment. In Proceedings of the ACM-SIAM Symposium on Discrete Algorithms.

Lazer, D., et al (2009). Life in the network: the coming age of computational social science. Science, 323(5915), 721.

Li, A., Cornelius, S. P., Liu, Y. Y., Wang, L., \& Barabási, A. L. (2017). The fundamental advantages of temporal networks. Science, 358(6366), 1042-1046.

Lupton, D. (2014). Digital sociology. Routledge.

Marres, N. (2017). Digital sociology: The reinvention of social research. John Wiley \& Sons.

Mehler, A., Pustylnikov, O., \& Diewald, N. (2011). Geography of social ontologies: Testing a variant of the Sapir-Whorf Hypothesis in the context of Wikipedia. Computer Speech \& Language, 25(3), 716-740.

Menini, S., Sprugnoli, R., Moretti, G., Bignotti, E., Tonelli, S., \& Lepri, B. (2017). RAMBLE ON: Tracing Movements of Popular Historical Figures. In Proceedings of the Software 
Demonstrations of the 15th Conference of the European Chapter of the Association for Computational Linguistics (pp. 77-80).

Michel, J.-B. et al. (2011) Quantitative Analysis of Culture Using Millions of Digitized Books. Science 331, 176-182.

Miller, D. \& Slater, D. (2000). The Internet: An ethnographic approach. Oxford: Berg.

Murray, C. (2003) Human Accomplishment. Harper Collins.

Nemoto, K., \& Gloor, P. A. (2011). Analyzing cultural differences in collaborative innovation networks by analyzing editing behavior in different-language Wikipedias. ProcediaSocial and Behavioral Sciences, 26, 180-190.

Orton-Johnson, K., \& Prior, N. (Eds.). (2013). Digital sociology: Critical perspectives. Springer.

Overell, S. E., \& Rüger, S. (2011). View of the world according to Wikipedia: Are we all little Steinbergs? Journal of Computational Science, 2(3), 193-197.

Page, L., Brin, S., Motwani, R. \& Winograd, T. (1999) The PageRank Citation Ranking: Bringing Order to the Web. Technical Report. Stanford InfoLab.

Pentland, A. (2014). Social Physics. How Good Ideas Spread - The Lessons from a New Science. New York: Penguin.

Pentland, A., \& Heibeck, T. (2010). Honest signals: how they shape our world. MIT press.

Popescu, A. \& Grefenstette, G. (2010). Spatiotemporal mapping of Wikipedia concepts. Proceedings of the 10th annual joint conference on Digital libraries, 129-138.

Reznik, I., \& Shatalov, V. (2016). Hidden revolution of human priorities: An analysis of biographical data from Wikipedia. Journal of informetrics, 10(1), 124-131.

Rochat, Y. (2009). Closeness centrality extended to unconnected graphs: The harmonic centrality index (No. CONF).

Rogers, R. (2013). Digital Methods. London: MIT Press.

Roll, U. et al (2016). Using Wikipedia page views to explore the cultural importance of global reptiles. Biological conservation, 204, 42-50.

Ronen, S., Gonçalves, B., Hu, K. Z., Vespignani, A., Pinker, S., \& Hidalgo, C. A. (2014). Links that speak: The global language network and its association with global fame. Proceedings of the National Academy of Sciences, 111(52), E5616-E5622.

Saramäki, J., Kivelä, M., Onnela, J. P., Kaski, K., \& Kertesz, J. (2007). Generalizations of the clustering coefficient to weighted complex networks. Physical Review E, 75(2), 027105.

Schäfer, M. T., \& Van Es, K. (Eds.). (2017). The datafied society: Studying culture through data. Amsterdam University Press.

Schich, M. et al. A network framework of cultural history. Science, 345, 558-562 (2014).

Shane-Simpson, C., \& Gillespie-Lynch, K. (2017). Examining potential mechanisms underlying the Wikipedia gender gap through a collaborative editing task. Computers in Human Behavior, 66, 312-328.

Skiena, S., \& Ward, C. B. (2013). Who's Bigger? Where Historical Figures Really Rank. Cambridge University Press.

Venturini, T., Bounegru, L., Gray, J., \& Rogers, R. (2018). A reality check (list) for digital methods. New media \& society, 1461444818769236.

Wickham, H. (2016). rvest: Easily Harvest (Scrape) Web Pages. $R$ package version 0.3.2. Retrieved from https://CRAN.R-project.org/package=rvest

Yu A. Z., Ronen, S., Hu, K., Lu, T., \& Hidalgo, C. A (2016). Pantheon 1.0, a manually verified dataset of globally famous biographies. Scientific Data 2:150075.

Zook, M., Dodge, M., Aoyama, Y., \& Townsend, A. (2004). New digital geographies: Information, communication, and place. In Geography and Technology (pp. 155-176). Springer, Dordrecht. 


\section{Annex}

Occupational domain recoding (variable 'domain2'):

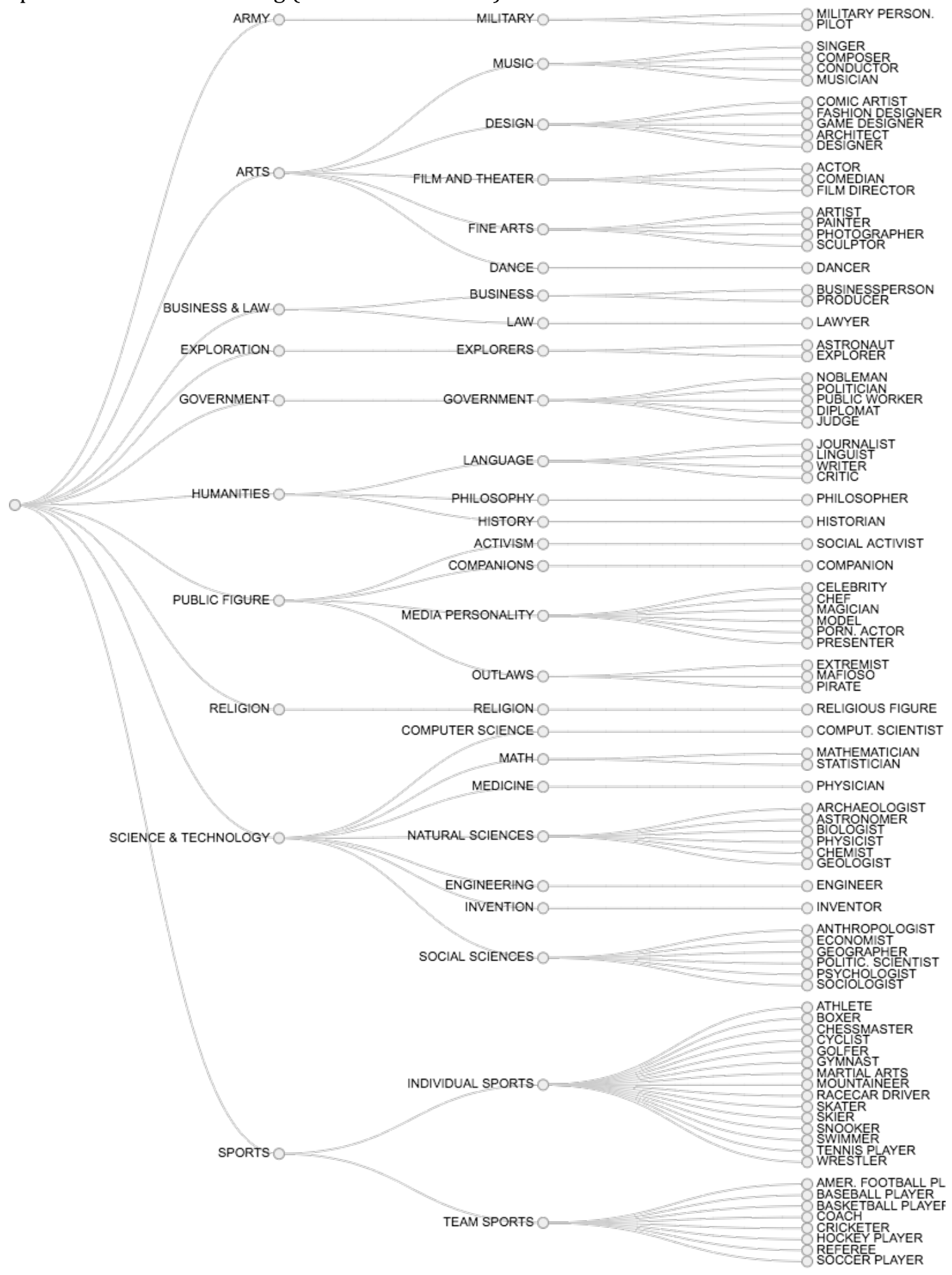

